\title{
The Research of Financial Futures Risk Monitoring based on CEP Technology
}

\author{
Shanshan WANG, Yun CHEN, Qiwei SUN \\ Shanghai key laboratory of financial information technology, School of information management and \\ engineering in Shanghai University of finance and economics, Shanghai, China
}

\begin{abstract}
The construction of financial futures risk monitoring system based on the complex transaction processing Sybase ESP oriented financial futures market requirements can greatly enhance the performance of futures transaction risk. This paper mainly introduce the main technical framework of financial futures risk monitoring system, the function of each module and the system characteristics
\end{abstract}

KEYWORD: Financial futures risk monitoring system; Complex transaction processing; Sybase ESP

\section{INTRODUCTION}

Financial futures has become one of the main trading products in the international financial market, however, if used improperly will cause huge risk in the function of risk aversion and price discovery. Therefore, only the maintenance of healthy and stable operation of the financial futures market can make it give full play to the function.

For China's Shanghai and Shenzhen 300 stock index futures market have achieved full electronic trading and settlement, the stock index futures market trading volume and position volume increased rapidly in recent years. Especially the domestic futures market automated trading has developed rapidly, which has advantages in terms of small single trust, large trust inventory, etc. At the same time under the background of financial futures innovation promotion, foreign exchange futures, stock index option will also be launched and the number of transactions and market participants will increase rapidly. The explosive growth of financial futures market volume will bring unknown challenge to risk monitoring tasks.

At present, the futures market risk monitoring system is mainly based on traditional data processing technology based, not enough to support high-speed, real-time monitoring. Complex transaction processing (CEP, Complex Event Processing) has the characteristics of strong data processing capability and short latency, and it can also provide a complete development environment, which can facilitate the development and deployment of the algorithm and the strategy.

Based on the applicatißon of complex transaction processing technology and abnormal trading behavior characteristics research, the system can be applied in the monitoring of market, entrusted funds, positions and so on, aiming to find the customer risk in time, and then help to decide to take different treatment measures, by obtaining the real-time market data through the trading system interface.

\section{SYSTEM}

\subsection{System architecture}

Futures risk monitoring system consists of four parts, the overall system architecture is shown in Figure1.

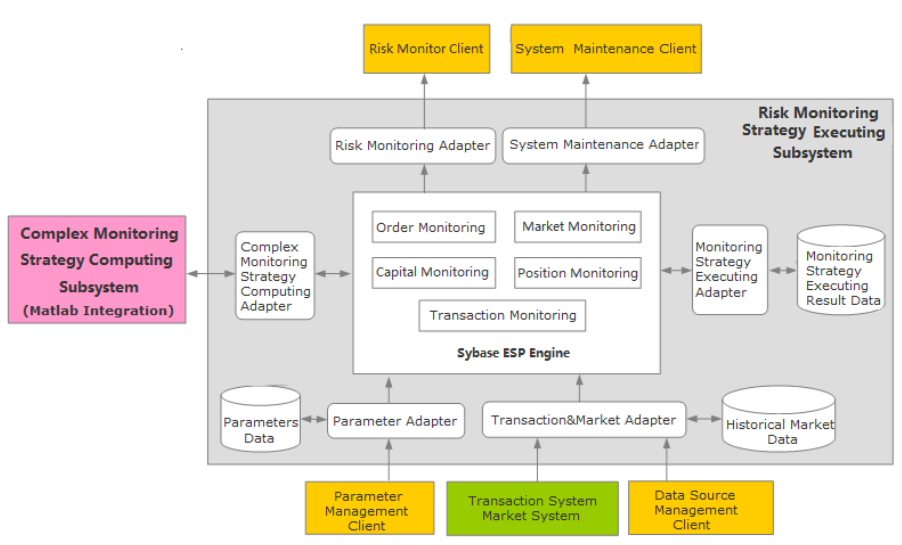

Figure1. System architecture

(1) Monitoring Strategies Executing Subsystem

The subsystem based on the real-time market data, executes risk monitoring strategies and output the risk customers according to the predefined threshold. The subsystem is composed of the following parts. 


\section{1) Trading \& Market Adapter}

This model accepts and initially filters source data from each member's transaction platform, to convert it to the format ESP engine requires.

2) Parameter Adapter

This model takes charge of transforming parameters defined by members to the format ESP engine requires, and loading global configurations during system boots up. And it records data from transaction system according to execution time, to provide historical data for replay transactions.

3) Complex Strategy Computing Adapter

It is responsible for data conversion between risk monitoring strategy computing subsystem and complex risk monitoring strategy computing subsystem, and maintains the network connection.

4) Strategy Executing Adapter

Taking into account of querying historical risk monitoring events, strategy execution data calculated by ESP engine will be stored into database by this model.

5) Risk Monitoring Adapter

It subscribes strategy execution data through ESP SDK, and sends it to external clients which listen on this. It also maintains connections with clients in account authentication and exceptions handling, etc.

6) System State Adapter

It collects states of ESP engine and other adapters, and sends them to the "system monitoring and maintenance client", to help administrator to analysis and manually fix exceptions during system runtime.

(2) Complex Risk Monitoring Strategy Computing Subsystem

It bases on professional mathematics software, such as Matlab, to accomplish several complex computing of monitoring, and sends the result to ESP engine for further operations.

\section{(3) External Data Source}

External data source mainly comes from trading and market system, such as FEMAS, Shanghai and Shenzhen stock market, etc, to provide real-time trading and market data for risk monitoring.

(4) External Controlling System

1) Parameter Management Client

It mainly helps to configure the risk monitoring relevant parameters, including: connection parameters of external system; business parameters, such as bond and fee rate; kinds of monitoring thresholds, etc.

2) Risk Monitoring Client

It shows results of strategy and warns administrator to manually fix if there's any exception. And it can classify the monitoring for exchange, clearing member and trading member.

3) Data Source Management Client

It helps on management of data source, to make risk monitor based on data from different trading and market system. In order to determine the rationality of parameter value, the user can replay the transaction based on historical data. The historical data is extracted according to the risk monitoring strategic requirements and sent to ESP engine according to the monitoring strategy execution logic.

4) System Monitoring \& Maintenance Client

It takes charge of monitoring states of system models, warning administrator to manually fix exceptions when they arise.

\subsection{Mode of risk monitoring strategy execution}

The risk monitoring system can handle real-time trading data, historical trading data and market indexes, etc, based on ESP. With ESP engine, there are three steps to execute risk monitoring strategy.

(1) Basic Data Layer

This layer simply manages data from source system and transform it into the basic data flow, which is used for following execution and subscription. It mainly provides data of market, trading, position, capital, parameters and so on, and also could be expended according to business requirements.

(2) Business Event Layer

Business event layer accepts data from basic data layer and calculate risk monitoring indexes, including trust, transaction, position, capital, market and so on, according to the risk monitoring rules. The result calculated will be output as data flow, or published for subscription from external system. This layer expands from business perspective, increases several business features horizontally, and achieves business process on different levels vertically.

(3) Risk Disclosure Layer

This lay accepts data from business event layer and compares it with pre-defined risk monitoring thresholds to discover risk in transaction process.

Figure 2 takes position proportion as example, to present execution process of risk monitoring strategy, based on ESP engine.

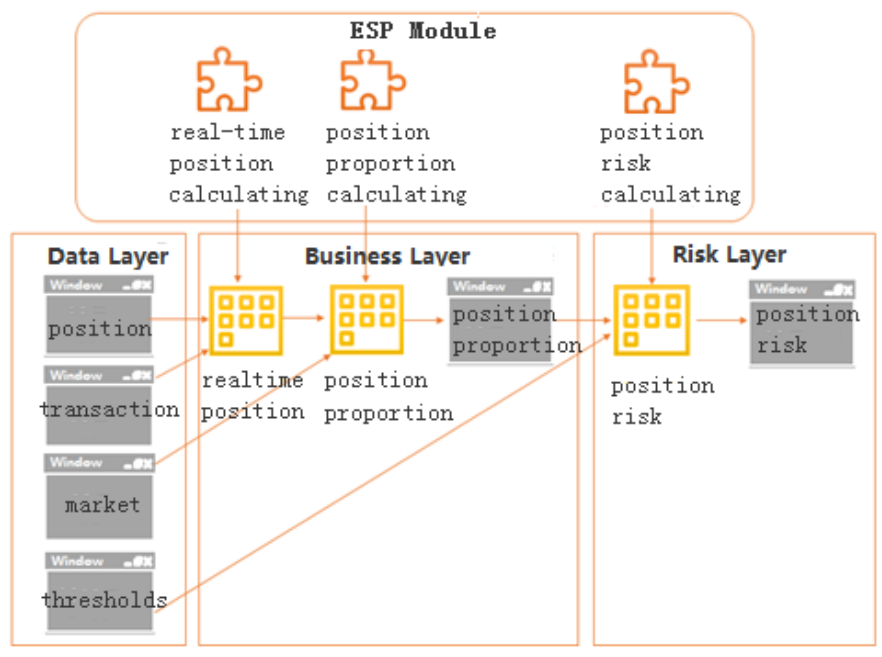

Figure2. Position proportion risk monitoring execution process 


\subsection{System Features}

Based on CEP technology, futures risk monitoring system has following features:

(1) Function: system can support typical risk monitoring based on market flow and trading flow; support pre-defined and flexible monitoring rules and thresholds; support replay based on historical trading and market data.

(2) Privilege: risk monitoring can be graded for exchange, clearing member and trading member; according to the demand of management, monitoring objects and parameters could be customized.

(3) Performance: system can support 1 million customers, 30,000 entrust and transaction per second as peak performance.

(4) Architecture: system takes modular design good for various of business expansion, because of its low coupling and high cohesion; original architecture, modules and source code could be reused to rapidly to achieve additional requirements.

\section{SUMMARY}

At present risk monitoring system has been very popular, but the performance of existing production is not good enough to support high throughput processing on real-time monitoring. Under this circumstance, a powerful, stable and flexible risk monitoring system, based on CEP technology, is needed. It could make futures risk monitoring more and more intelligent, from simply query mode to automatic warning mode, from post passive monitoring to active monitoring. By the way, it also enhances risk management of market development across market supervision, etc. However, system construction is very complex and requires many efforts and various resources. The system mentioned here, has already been basically completed, but is still pending to production environment, for its realtime performance, flexibility and security still needs to be improved.

\section{REFERENCES}

[1] Lifeng Xiang \& Jianlin Sun 2010. Research of transaction based on complex event processing. Computer Science Application and Software.

[2] Suhothayan S. \& Gajasinghe K. 2011. A second look at complex event processing architectures, Proceedings of the 2011 ACM workshop on Gateway computing environments. ACM.

[3] Introduce of FEMAS Trade Platform API.

[4] Sybase software, Programmers Guide Sybase Event Stream Processor.

[5] Sybase software, Programmers Reference Sybase Event Stream Processor. 